Aim of the study: To assess resource utilization and costs of treatment with lanreotide AUTOGEL $120 \mathrm{mg}$ (ATG120) administered as part of routine acromegaly care in Poland.

Material and methods: A multicentre, non-interventional, observational study on resource utilization in Polish acromegalic patients treated with ATG120 at 4 weeks or extended (> 4 weeks) dosing interval. The study recruited adult acromegalic patients treated medically for $\geq 1$ year including at least 3 injections of ATG120. Data on dosing interval, aspects of administration, and resource utilization were collected prospectively during 12 months. Costs were calculated in PLN from the public health-care payer perspective for the year 2013.

Results: 139 patients were included in the analysis. Changes in dosing regimen were reported in 14 (9.4\%) patients. Combined treatment was used in 11 (8\%) patients. Seventy patients $(50 \%)$ received ATG120 at an extended dosing interval; the mean number of days between injections was 35.56 (SD 8.4). ATG120 was predominantly administered in an out-patient setting $(77 \%)$, by health-care professionals (94\%). Mean time needed for preparation and administration was 4.33 and $1.58 \mathrm{~min}$, respectively, mean product wastage $-0.13 \mathrm{mg}$. Patients were predominantly treated in an out-patient setting with 7.06 physician visits/patient/ year. The most common control examinations were magnetic resonance imaging of brain and brain stem (1.36/ patient/year), ultrasound of the neck (1.35/patient/year), GH (1.69/patient/ year), glycaemia (1.12/patient/year), IGF-1 (0.84/patient/year), pituitary-thyroid axis hormone levels assessment (TSH-0.58/patient/year, T4-0.78/patient/ year). There were 0.43 hospitalizations/patient/year. For direct medical costs estimated at PLN 50 692/patient/ year the main item was the costs of ATG120 (PLN 4103.87/patient/month; 97\%). The mean medical cost, excluding pharmacotherapy, was PLN 1445/patient/year (out-patient care $-49 \%$, hospitalization $-23 \%$, diagnostics/laboratory tests - 28\%).

Conclusions: These results represent the current use of ATG120 in the population of Polish acromegalic patients in a realistic clinical setting. Findings that $50 \%$ of patients could be treated with dose intervals of longer than 28 days support the potential of ATG120 to reduce the treatment burden.

Key words: acromegaly, lanreotide, clinical study, costs of treatment

Contemp Oncol (Pozn) 2013; 17 (5): 460-465 DOI: $10.5114 /$ wo.2013.38805

\section{Assessment of real-world usage of lanreotide AUTOGEL 120 in Polish acromegalic patients - results from the prospective 12-month phase of Lanro-Study}

\author{
Ewa Orlewska ${ }^{12}$, Beata Kos-Kudta ${ }^{3}$, Jerzy Sowiński ${ }^{4}$, Krzysztof Sworczak $^{5}$, \\ Wojciech Zgliczyński ${ }^{6}$ \\ oraz grupa badaczy badania Lanro-Study: Elżbieta Andrysiak-Mamos', \\ Anna Babińska ${ }^{5}$, Agata Bałdys-Waligórska ${ }^{8}$, Elżbieta Bandurska-Stankiewicz, \\ Krzysztof Błaut ${ }^{5}$, Paweł Bolko ${ }^{10}$, Wanda Foltyn ${ }^{3}$, Danuta Jakubczyk ${ }^{11}$, \\ Aleksandra Jawiarczyk-Przybyłowska ${ }^{12}$, Roman Junik ${ }^{13}$, Olga Juraniec ${ }^{13}$, \\ Ewelina Lewkowicz ${ }^{8}$, Anna Lewczuk ${ }^{5}$, Beata Matyjaszek-Matuszek ${ }^{14}$, \\ Krzysztof Michałek ${ }^{4}$, Sławomir Mucha ${ }^{15}$, Renata Orłowska-Florek ${ }^{16}$, \\ Marta Peszel-Barlik ${ }^{17}$, Sławomir Pynka ${ }^{18}$, Violetta Rosiek ${ }^{3}$, Marek Ruchała ${ }^{4}$ \\ Joanna Rutkowska9 , Julia Słynko-Krzyzostaniak', Agnieszka Stefańska ${ }^{8}$, \\ Janusz Strzelczyk ${ }^{3}$, Anhelli Syrenicz ${ }^{7}$, Małgorzata Trofimiuk-Müldner ${ }^{8}$, \\ Joanna Waligórska-Stachura ${ }^{4}$, Ryszard Waśko ${ }^{4}$, Przemysław Witek ${ }^{19}$, \\ Danuta Zalewska-Rydzkowska ${ }^{13}$, Piotr Zdunowski ${ }^{6}$, Anna Zemczak ${ }^{3}$
}

${ }^{1}$ Centre for Pharmacoeconomics, Warsaw, Poland

2Faculty of Health Sciences, Jan Kochanowski University, Kielce, Poland

${ }^{3}$ Division of Endocrinology, Department of Pathophysiology and Endocrinology, Silesian Medical University, Katowice, Poland

${ }^{4}$ Chair and Department of Endocrinology, Metabolism and Internal Diseases, Poznan University of Medical Sciences, Poznan, Poland

${ }^{5}$ Endocrinology and Internal Diseases Department, Medical University, Gdansk, Poland ${ }^{6}$ Endocrinology Department Medical Center of Postgraduate Education, Warsaw, Poland 7Endocrinology, Metabolic Diseases and Internal Medicine Department, Pomeranian Medical University, Szczecin; ${ }^{8}$ Endocrinology Department, University Hospital, Krakow; ${ }^{9}$ Endocrinology, Diabetology and Internal Medicine Department, University of Warmia and Masuria, Olsztyn; ${ }^{10}$ Clinical Practice, Plewiska; ${ }^{11}$ Endocrinology, Diabetology and Internal Medicine Department, Medical Academy University, Bialystok; ${ }^{12}$ Chair and Department of Endocrinology and Diabetology, Wroclaw Medical University, Wroclaw, ${ }^{13}$ Endocrinology and Diabetology Department, University Hospital, Bydgoszcz,

${ }^{14}$ Endocrinology and Internal Medicine Department, Public Clinical Hospital, Lublin; ${ }^{15}$ Endocrinology Office, Lodz; ${ }^{16}$ Endocrinology Clinic, Hospital no 2, Rzeszow;

${ }^{17}$ Endocrinology Clinic, Tarnowskie Gory; ${ }^{18}$ Diabetological and Internal Medicine Clinic, Szczecin; ${ }^{19}$ Department of Endocrinology and Isotope Therapy, Military Institute of Medicine, Warsaw

\section{Introduction}

Somatostatin analogues (SA) are the most commonly used forms of medical therapy for acromegaly. Currently used SA (octreotide and lanreotide) are available as long-acting preparations with product-specific formulations and means of administration [1]. The pharmacokinetic profile of lanreotide AUTOGEL (Ipsen Pharma) is different from that of octreotide LAR and is characterized by a rapid but moderate initial increase within the first 12 hours followed by a gradual decline with an apparent elimination half-life of $\sim 22$ days [2]. The results of studies [3-5] demonstrated that ATG120 administered every 4, 6, or 8 weeks can provide effective biochemical control of acromegaly, with a safety profile similar to that seen with a traditional 4-week dosing interval. 
The aim of this study is to evaluate over the 12-month prospective follow-up resource utilization and costs of treatment of acromegalic patients in Poland with ATG120 when administered as part of routine clinical practice. This type of study provides evidence on the use of a drug in a realistic setting and can be utilized as a vehicle for addressing multiple post-approval objectives, e.g. optimizing product usage, documenting cost-effectiveness, and identifying best practice. Results of this study will allow the decision maker to make informed choices based on evidence and will contribute to efficient resource allocation.

\section{Material and methods}

This study was conducted in accordance with the Declaration of Helsinki [6] and the International Ethical Guidelines for Epidemiological Studies, CIOMS, Feb 2008 [7]. This study also followed the recommendations of the International Epidemiological Association Guidelines for Proper Conduct in Epidemiologic Research (GEP) - Nov 2007 [8], and the International Society for Pharmacoepidemiology (ISPE) Guidelines for Good Pharmacoepidemiology Practices (GPP) [9]. As a non-interventional study this study falls outside the scope of the EU Directive 2001/20/EC, the EU Directive 2005/28/EC and ICH-GCP guidelines. This study complies with the EU Directive 95/46/EC of the European Parliament and of the Council of 24 October 1995 on the protection of individuals with regard to the processing of personal data and on the free movement of such data. All patients gave written informed consent before entering the study.

\section{Patients}

139 patients aged $22-86$ years (mean 51.5 ) with a clinical diagnosis of acromegaly, treated medically for $\geq 1$ year including at least 3 injections of ATG120, were included in this study.

Patients were excluded if they actively participated in any interventional or any other non-interventional acromegaly clinical study. Previous participation in an observational/PMS study of another somatostatin analogue was not an exclusion criteria. Patients were recruited in 36 centres between 29 October 2010 and 31 March 2012.

\section{Methods}

This was a national, multicentre, non-interventional, observational, prospective study to assess resource utilization and costs in the population of Polish acromegalic patients treated with ATG120 at 4 weeks or extended (6-8 weeks) dosing interval. As the study is non-interventional, the decision to prescribe the product was taken prior to, and independently from the decision to enrol the subject. This decision was made in accordance with routine clinical practice at the centre concerned. Treatment monitoring, dose adjustment and all other medical decisions were made at the responsible physician's discretion, according to the routine practice at the study site. Patients were included in the study during routine out-patients visits. All sites that treat patients meeting the study inclusion criteria were selected and contracted. Site selection was based on the ability to collect the data in electronic format, motivation to participate and fulfilling all requirements of the protocol. Relevant data collected prospectively during 12 months were captured in an electronic Case Report Form (eCRF). Data on resource utilization consisted of dosing interval and aspects of administration, hospitalizations, outpatient visits, laboratory/diagnostics tests. Cost evaluation for the study was performed from the perspective of the public payer, e.g. the National Health Fund, in 2013. Costs were assessed using a micro-costing approach. Unit cost multipliers were applied to the quantity of each type of resources consumed. Mean cost per patient in the 1-year time horizon resulted from the total cost divided by the number of patients included. The valuation of hospitalizations, out-patient consultations and laboratory/diagnostic tests was based on accounting information provided by the administrative departments of centres involved in the study, and the cost of pharmacotherapy was calculated based on the reimbursement list in force from $1^{\text {st }}$ July 2013 [10]. Costs of medicines, diagnostics and treatment received by patients in an in-patient setting were included in hospitalization costs which are reflected in the score of particular DRGs (diagnosis-related groups).

The endpoints of the study included:

- percentage of patients treated with ATG120 every 4, 6 and 8 weeks during the 12-month prospective observation,

- resources utilization, related workload and costs per patient/month or year of treatment during the 12-month prospective observation.

Given the exploratory nature of the study, no formal statistical analysis was performed.

\section{Results}

139 patients aged $22-86$ years (72\% female, $n=100$ ) were included in the analysis. The baseline demographic characteristics of the study population are summarized in Table 1.

\section{Pharmacotherapy}

During the 12-month prospective phase of the study 70 patients (50\%) received ATG120 at an extended dosing interval ( $>4$ weeks); the mean number of days between injections was 35.56 (SD 8.4). In 125 patients (90\%) the dosing

Table 1. Demographic and baseline characteristics

\begin{tabular}{|lc|}
\hline Variable & Population $(n=139)$ \\
\hline Age - mean (SD), years & $51.5(14.0)$ \\
\hline Weight - mean (SD), kg & $82.95(17.8)$ \\
\hline BMI - mean (SD), kg/m² & $29.51(5.5)$ \\
\hline $\begin{array}{l}\text { Gender } \\
\text { male, } n \text { (\%) } \\
\text { female, } n \text { (\%) }\end{array}$ & $39(28)$ \\
\hline Pituitary surgery, $n$ (\%) & $100(72)$ \\
\hline Radiotherapy, $n$ (\%) & $107(77)$ \\
\hline $\begin{array}{l}\text { Length - mean (SD) length of treatment } \\
\text { with ATG120, months }\end{array}$ & $26(19)$ \\
\hline
\end{tabular}


regimen of ATG120 remained unchanged. In this subpopulation injections were indicated every 4, 6 and 8 weeks in $67.5 \%, 19.8 \%$ and $8 \%$ of patients, respectively, but in practice were administered every 4 weeks in $53.2 \%$, every 5 weeks in $18.3 \%$, every 6 weeks in $17.5 \%$, every 7 weeks in $4.8 \%$, every 8 weeks in 3.2\%, and every 9 weeks in $2.4 \%$ of patients. Changes in dosing regimen of ATG120 were reported in 14 patients: 8 patients had the dose frequency extended, in 5 patients the frequency increased, and in 1 patient treatment was switched from ATG120 injected every 4 weeks to octreotide LAR $20 \mathrm{mg}$ every 4 weeks and later octreotide LAR $30 \mathrm{mg}$ every 4 weeks.

ATG120 was predominantly administered in an outpatient setting (77\% of injections), by health-care profes- sionals (94.5\% of injections) (Table 2). Mean time needed for preparation and administration was 4.33 min (SD 2.8) and $1.58 \mathrm{~min}$ (SD 1.24), respectively, mean product wastage $-0.13 \mathrm{mg}$.

\section{Other resources}

The tables below present the annual consumption of resources for the treatment of acromegalic patients with lanreotide AUTOGEL. The mean number of hospitalizations and out-patient consultations is presented in Table 3. Furthermore, the diagnostic procedures requested by specialists and general practitioners are presented in Table 4 and 5, respectively. Data on the mean number of such procedures provides information about the diagnostic regimens followed by pri-

Table 2. Administration of lanreotide AUTOGEL (setting, person, who administer lanreotide AUTOGEL)

\begin{tabular}{|c|c|c|c|c|c|c|}
\hline & & \multicolumn{4}{|c|}{ Person who administered lanreotide AUTOGEL } & \multirow[t]{2}{*}{ Total } \\
\hline & & Physician & Care-giver & Patient & Nurse & \\
\hline \multirow{4}{*}{$\begin{array}{l}\text { Setting of } \\
\text { administration }\end{array}$} & House & - & 15 & 12 & 3 & 30 \\
\hline & Out-patient setting & 169 & 4 & - & 857 & 1030 \\
\hline & In-patient setting & 26 & 3 & 1 & 241 & 271 \\
\hline & Lack of data & - & 3 & - & - & 3 \\
\hline Total & & 195 & 25 & 13 & 1101 & 1334 \\
\hline
\end{tabular}

Combined treatment was used in 11 (8\%) patients. 5 patients received SA and bromocriptine, 5 patients - SA and cabergoline and in 1 patients - SA and pegvisomant. Mean time (SD) of treatment was 204.37 (123.21), 301.8 (34.61) and 267 days for bromocriptine, cabergoline and pegvisomant, respectively.

Table 3. Annual consumption of resources: hospitalizations and out-patient consultations

\begin{tabular}{|lccc}
\hline Resource item & User, $n(\%)$ & Average number/ patient/year & Average number/user/year \\
\hline Hospitalizations & $89(64.0$ days $)$ & $0.43(1.7$ days $)$ & $1.61(6.6$ days $)$ \\
endocrinology ward & $41(29.5)$ & $0.29(1.2$ days $)$ & $1.17(3.9$ days $)$ \\
surgery ward & $8(5.7)$ & $0.057(0.3$ days $)$ & $1(5.9$ days $)$ \\
internal medicine ward & $5(3.6)$ & $0.036(0.1$ days $)$ & 7.8 days $)$ \\
Out-patient consultations & $139(100)$ & 7.06 & 3.3 \\
endocrinologist & $139(100)$ & 3.3 & 3.43 \\
other specialist & $90(65)$ & 2.22 & 2.93 \\
primary care physician & $71(51)$ & 1.5 & \\
\hline
\end{tabular}

Table 4. Annual consumption of resources: diagnostic procedures

\begin{tabular}{lccc} 
& $N$ & Average number per patient annually & Average number per user annually \\
\hline $\begin{array}{l}\text { Magnetic resonance imaging of: } \\
\text { brain and brain stem }\end{array}$ & 87 & 0.63 & 1.36 \\
\hline $\begin{array}{l}\text { Ultrasound examination of: } \\
\text { neck }\end{array}$ & 67 & & \\
abdomen and retroperitoneum & 31 & 0.48 & 1.35 \\
heart & 12 & 0.22 & 1.11 \\
peripheral vascular system & 1 & 0.09 & 1 \\
X-ray examination of: & & 0.01 & 1 \\
thorax & 17 & & 1.13 \\
extremities and pelvis & 9 & 0.12 & 1.29 \\
soft tissues of abdomen & 1 & 0.07 & 1 \\
\hline Bone mineral density & 4 & 0.01 & 1 \\
\hline Endoscopy: & & 0.03 & 1 \\
colonoscopy & 6 & & 1 \\
gastroscopy & 1 & 0.04 & \\
\end{tabular}


Table 5. Annual consumption of resources: diagnostic tests

\begin{tabular}{|c|c|c|c|}
\hline Type of test & No. of tests & Average number per patient annually & Average number per user annually \\
\hline Somatotropin (GH) & 235 & 1.69 & 2.26 \\
\hline Insulin-like growth factor 1 (IGF-I) & 117 & 0.84 & 1.01 \\
\hline Glucose & 155 & 1.12 & 1.94 \\
\hline Glycated haemoglobin (HbA1C) & 64 & 0.46 & 1.78 \\
\hline Blood tests & 59 & 0.42 & 1.51 \\
\hline Asparaginian aminotransferase (AspAT) & 48 & 0.35 & 1.60 \\
\hline Alanine aminotransferase (AIAT) & 60 & 0.43 & 1.58 \\
\hline Bilirubin & 43 & 0.31 & 1.39 \\
\hline Creatinine & 50 & 0.36 & 1.47 \\
\hline Uric amid & 13 & 0.09 & 1.08 \\
\hline Triglyceride & 35 & 0.25 & 1.30 \\
\hline Total cholesterol & 59 & 0.42 & 1.48 \\
\hline Low-density lipoprotein (LDL) & 20 & 0.14 & 1.00 \\
\hline High-density lipoprotein (HDL) & 29 & 0.21 & 1.45 \\
\hline Very-low-density lipoprotein (VLDL) & 28 & 0.20 & 1.47 \\
\hline Insulin & 7 & 0.05 & 1.00 \\
\hline Iron & 1 & 0.01 & 1.00 \\
\hline Thyrotropin (TSH) & 81 & 0.58 & 1.60 \\
\hline Free thyroxine (fT4) & 108 & 0.78 & 1.66 \\
\hline Triiodothyronine (fT3) & 21 & 0.15 & 1.17 \\
\hline Anti-thyroid antibody & 1 & 0.01 & 1.00 \\
\hline
\end{tabular}

mary and specialist care physicians and thus constitute added value of this analysis.

Patients were predominantly treated in an out-patient setting with 7.06 physician visits/patient/year. The most common control examinations were magnetic resonance imaging of the brain and brain stem (1.36/patient/year), ultrasound of the neck (1.35/patient/year), GH level (1.69/patient/year), glycaemia (1.12/patient/year), IGF-1 level (0.84/patient/year), pituitary-thyroid axis hormone levels (TSH - 0.58/patient/year, T4 $-0.78 /$ patient/year). The number of hospitalizations was 0.43/patient/year (1.7 days/patient/year); the majority were hospitalizations in an endocrinology ward (0.29/patient/year; 1.2 days/patient/year).

\section{Costs}

The average direct medical cost per patient over a 12-month period was estimated at PLN 50692 (Table 6). Treatment with ATG120 accounts for the largest share of total costs. The cost calculated based on reimbursement status and retail price of ATG120 in force from 1 July 2013 [9] was PLN 4103.87/ patient/month (Table 7) and constituted $97 \%$ of the mean direct medical cost. For direct medical cost without pharmacotherapy (PLN 1445/patient/year) the main items were: out-patient care (PLN 706/patient/year, 49\%), hospitalization (PLN 329/patient/year, 23\%), diagnostics (PLN 291/patient/year, 20\%) and laboratory tests (PLN 119/patient/year, 8\%).
Table 6. Average direct medical cost of acromegalic patient's treatment: detailed breakdown

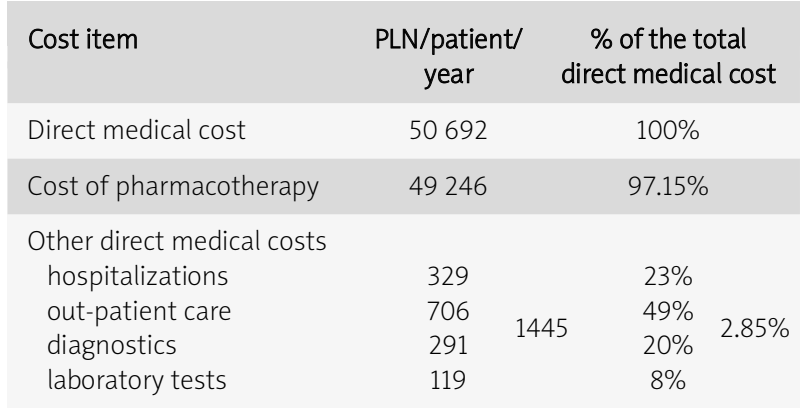

\section{Discussion}

This study presents the current use of ATG120 in the population of Polish acromegalic patients in a realistic clinical setting. The value of this type of study and usefulness of the results for medical decision makers is highly dependent upon the design of the study, methodology applied and population included. It should be noted that this study is an observational non-interventional study and there are no protocol-driven costs. The prospective design reduces recall bias, considered a weakness of retrospective trials. As the purpose of the study was to include all relevant costs within a health care system, a micro-costing approach was chosen. This method tracks resource consumption as it 
Table 7. Cost of lanreotide AUTOGEL $120 \mathrm{mg}(n=138)$

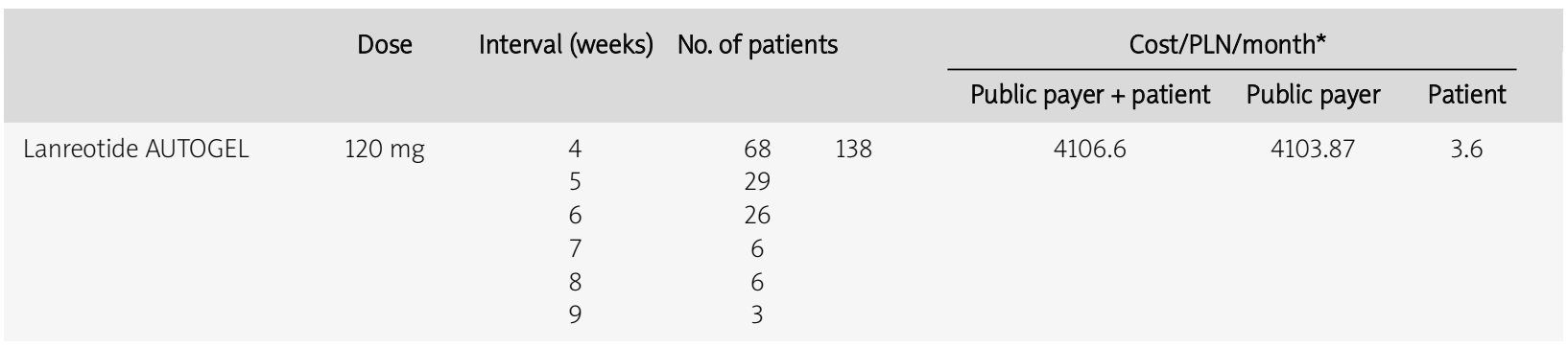

Retail and reference price of lanreotide AUTOGEL $120 \mathrm{mg}$ - PLN 4874.07/pack, fixed-fee payment (lump sum payment) [10]

occurs, and the level of precision obtainable in this kind of study is exemplary.

The study population, coming from 36 centres in different Polish regions, represents a wide cross-section of acromegalic patients in our country and seems to be representative. The demographic and clinical characteristics of this population do not differ from those described in acromegalic samples in other countries [11]. The only exception was higher prevalence of acromegaly in women than in men and this observation constitutes added value of this study.

Every costing study contains some degree of imprecision and methodological controversy. It concerns mainly valuation of resources used. Unit cost multipliers applied to the quantity of medical resources in the presented study were derived from information provided by the administrative departments of centres involved in the study. It is worth noting that some basic diagnostic procedures usually do not affect the cost of treatment as their cost is included in the cost of out-patient consultation or hospitalization. This is the reason that the costs were presented in the study in both aggregated and disaggregated form, allowing the decision maker to focus on particular items only.

Very limited economic data have been published so far concerning the cost of medical care in patients with acromegaly. The two economic studies retrieved in a very recently published review [12] address a comparison of disease burden between controlled and uncontrolled patient groups in Italian and Spanish settings $[13,14]$. In the Italian study, which enrolled 134 patients, total annual costs were reported to be higher in patients with uncontrolled disease (EUR 12,533) than in those with controlled disease (EUR 7968). Results from the Spanish study, which enrolled 11 patients, were reversed, because annual global treatment costs were higher in 6 patients with controlled disease (EUR 9874) versus 5 patients with uncontrolled disease (EUR 7072). This result might be explained by the difference in pharmacological treatment patterns: 3 of 6 uncontrolled patients were treated with bromocriptine, which is less expensive than SA. Both studies reported SA costs as a major cost driver alongside surgery, which is similar to our findings. We did not introduce the concept of controlled vs uncontrolled patient groups into our analysis because our primary aim was not to deliver a full assessment of the economic burden of acromegaly even though this topic is of high interest. We focus on treatment with ATG120 when administered as part of routine clinical practice.
Findings that $50 \%$ of patients could be treated with dose intervals longer than 28 days support the potential of ATG120 to reduce the treatment burden. The cost calculated based on reimbursement status and retail price of ATG120 in force from 1 July 2013 [10], i.e. PLN 4103.87/ patient/month, and was much lower than the price per pack, taking into account the prolonged intervals between injections in substantial proportion of treated patients. Short administration time, shown in our study and confirmed also by other study [15] might be important to take into consideration when comparing costly drugs which must be administered by injection.

This study was founded by Ipsen Poland Sp. z o.O.

\section{References}

1. Murray RD, Melmed S. A critical analysis of clinically available somatostatin analogue formulations for therapy of acromegaly. J Clin Endocrinol Metab 2008; 93: 2957-68.

2. Antonijoan RM, Barbanoj MJ, Cordero JA, et al. Pharmacokinetics of a new Autogel formulation of the somatostatin analogue lanreotide after a single subcutaneous dose in healthy volunteers. J Pharm Pharmacol 2004; 56: 471-6

3. Lucas T, Astorga R; Spanish-Portuguese Multicentre Autogel Study Group on Acromegaly. Efficacy of lanreotide Autogel administered every 4-8 weeks in patients with acromegaly previously responsive to lanreotide microparticles $30 \mathrm{mg}$ : a phase III trial. Clin Endocrinol (Oxf) 2006; 65: 320-6.

4. Lombardi G, Minuto F, Tamburrano G, et al. Efficacy of the new longacting formulation of lanreotide (lanreotide Autogel) in somatostatin analogue-naive patients with acromegaly. J. Endocrinol Invest 2009; 32: 202-9.

5. Gomez-Panzani E, Chang S, Ramis J, et al. Sustained biochemical control in patients with acromegaly treated with lanreotide depot 120 mg administered every 4 weeks or an extended dosing intervals of 6 to 8 weeks: a pharmacokinetic approach. Research \& Reports in Endocrine Disord 2012; 2: 79-84.

6. Declaration of Helsinki, 2008

7. International Ethical Guidelines for Epidemiological Studies, CIOMS, Feb. 2008.

8. International Epidemiological Association Guidelines for the Proper Conduct in Epidemiologic Research (GEP) - Nov 2007.

9. International Society for Pharmacoepidemiology (ISPE) Good PharmacoEpidemiological Practices (GPP) Guidelines, April 2007.

10. Obwieszczenie Ministra Zdrowia z dnia 24 czerwca 2013 r. w sprawie refundowanych leków, środków specjalnego przeznaczenia żywieniowego oraz wyrobów medycznych na dzień 1 lipca 2013. http://www.mz.gov.pl/wwwmz/index?mr=q491\&ms=383\&ml=pl\&mi $=383 \& m x=0 \& m t=\& m y=419 \& m a=32235$ ).

11. Melmed S. Medical Progress: acromegaly. N Engl J Med 2006; 355: 2558-73. 
12. Ben-Shlomo A, Sheppard MC, Stephens JM, Pulgar S, Melmed S. Clinical, quality of life and economic value of acromegaly disease control. Pituitary 2011; 14: 284-94.

13. Didoni G, Grottol S, Gasco V, et al. Cost-of-illness study in acromegalic patients in Italy. J Endocrinol Invest 2004; 27: 1034-9.

14. Luque-Ramírez M, Paramo C, Varela da Costa C, García-Mayor RV. Cost of management of invasive growth hormone secreting macroadenoma. J Endocrinol Invest 2007; 30: 541-545.

15. Schweinsberg K, Smith S, Kirshner LS. Ease of administration of somatostatin analogues, octreotide LAR versus lanreotide. Presented at 93rd annual meeting of the Endocrine Society, June 4-7, 2011, Boston, MA.

\section{Address for correspondence}

Ewa Orlewska MD, PhD

Centre for Pharmacoeconomics

Kremowa 13

02-969 Warsaw, Poland

e-mail: ewaorlewska@tlen.pl

Submitted: 13.09.2013

Accepted: 18.10.2013 